\title{
Evaluation of Some Medicinal Plants Diversity and Compendium Information on the Usage in Michika, Adamawa state
}

\author{
Isaac John Umaru ${ }^{1 *}$, Hauwa A. Umaru², Franklin John Umaru ${ }^{3}$, Kerenhappuch Isaac John³ ${ }^{3}$ Umaru \\ Maryam Ahmed Usman ${ }^{3}$ and Abdulrashid Mohammed ${ }^{3}$ \\ ${ }^{T}$ Department of Biochemistry, Adamawa State University of Mubi, Adamawa State Nigeria \\ ${ }^{2}$ Department Medical Biochemistry, Faculty of Basic Science, Collage of Medical Sciences Abubakar Tafawa Balewa \\ University, Bauchi State, Nigeria \\ ${ }^{3}$ Department of Agriculture, Moddibo Adama University of Technology, Yola Adamawa State, Nigeria
}

\section{*Corresponding Author}

Isaac John Umaru

\author{
Article History \\ Received: 10.06 .2020 \\ Accepted: 21.06 .2020 \\ Published: 25.06.2020
}

\begin{abstract}
The study assessed the diversity of the indigenous tree species and also produce of a compendium of information on the various medicinal uses of the indigenous tree species and their parts in Adamawa State, Michika local government seven districts; Bazza, Futu, Garta, Madzi, Michika, Nkafa, Zah. Sample plots method was used in the assessment of the indigenous tree species using questioner. All indigenous Medicinal plants information on ethonomedicinal uses was obtained through oral interviews and the through the questioners. Age group and occupation was considered such as; Local healer, Herbalist, Traditional birth attendance, experienced parents, patients and students were randomly selected for this purpose. Result of the findings indicated that 48 common indigenous medicinal plants species were encountered in all the seven districts inventoried in the study. Results further indicate that Acacia seyal, Parka biglobosa, Adonsonia digitata ,Khaya senegalensis, Ziziphus spina-christi, Azadirachta indica, Moringa oliefera, Ficusgnaphalocarpa, Mangifera indica, Cassipourea congoensis, Psidium guajava, Vitex doniana, Nuclea latifolia, Anacardium occidental, Nuclea latifolia, Carica papaya, Annona senegalensis, Citrus aurrantium, Eucalyptus indica, Tamarindus indica, Vernonia amygdalina, Cassia occidentalis, Citrus Paradise, Momordica charantia, Anogeissuss, leiocapus, Ficus vollis, Gemlin arborea, Borassus aethiopum, Abrus precatorius, Elaeophorbia drupifera, Artemisia maciverae, Vitellaria paradoxa, Zizipus Mauritania, Daniella oliverii, Anogeissus leiocarpa, Ficus sycomorus, Balanites aegyptiaca, Conorandus panados, Deterium microcarpum, and .Balanites aegytiaca were common to all the districts. While all other species were found in either three, two or one districts. Diversity Index community response on resource and ranking of medicinal plant in the seven districts indicate Nkafa and Zah was observed to have higher ranking. It was observed that the plant parts used for medicinal purposes are roots, barks, leaves, stems, Epiphyte etc. The formulation for medication include; boiling, soaking, etc. Methods of administration include, drinking, bathing, adding to food as spices and chewing. Among the ailments treated are dysentery, diarrhea, diabetes, Tuberculosis, Meningitis, Blood tonic, High blood Pressure, Worms, Typhoid Fever, Cancer, Malnutrition, Tumors, Wound Healing, Jaundice, Epilepsy and Rheumatism. It can thus be concluded that the sharp decrease in disease outbreak in the districts and villages was as to the increasing economic and health values placed on medicinal plants, documentation on ethnobatanical knowledge is a way to understand the use of different plant species to cure various ailments, diseases and means to conserve these natural resources. From the findings of the study the following recommendations are hereby made; the local people need to be trained, encouraged and supported on how to conserve and manage the medicinal plant species, Young generation need awareness to avoid negative impacts on the medicinal plants and associated knowledge in the area, hence, documentation of the medicinal plants of the area needs to continue.
\end{abstract}

Keywords: Evaluation, Medicinal Plants, Diversity, Compendium, Michika, Adamawa state.

Copyright @ 2020: This is an open-access article distributed under the terms of the Creative Commons Attribution license which permits unrestricted use, distribution, and reproduction in any medium for non commercial use (NonCommercial, or CC-BY-NC) provided the original author and source are credited. 


\section{INTRODUCTION}

The World Health Organization (WHO) defines traditional medicine to include a diversity of health practices, approaches, knowledge, and beliefs incorporating plant, animal, and/or mineral-based medicines; spiritual therapies; manual techniques; and exercises, applied singly or in combination to maintain well-being, as well as to treat, diagnose, or prevent illness [1].

Knowledge of traditional medicine is an integral part of the indigenous knowledge of local communities which according to Sithole is a complete body of knowledge, know-how and practices maintained and developed by the people, generally in rural areas, who have extended histories of interaction with the natural environment. This interaction sets understandings, interpretations and meanings that are part of a cultural complex [2].

Traditional medicine knowledge is that aspect of indigenous knowledge of people in local communities which relates to the use of plants and other natural resources in the treatment of health related conditions. From time immemorial, plants and its allied products has been used in the treatment of various ailments all over the world especially in local communities in developing countries. Traditional medicine knowledge begins with the study of local plants species to identify edible, medicinal and poisonous ones. Plant forms the main ingredients of medicine in traditional system of healing and has been the source of inspiration for several major pharmaceutical drugs [3].

In African continent, traditional medical practices date as far back as 4000 years. It was the sole medication for the health care in those days before the advent of modern medicine. Traditional medicine in this present era traditional medicine is becoming predominate of which most of the synthesized drugs have their bases from Traditional medicine [4].

Traditional medicine knowledge goes beyond knowledge of what plant species is used for treatment of a particular ailment. According to Nijar, to transform a plant into a medicine, one has to know not just the current specie but also its location, and possibly its local usage [5].

One of the basic features of traditional knowledge is that it is unwritten and exists in the minds of the local people. It is transmitted orally from one generation to another. TK plays a significant role in the lifestyle of the members of the local community and hence an essential resource for any human development process. They form the basis for decisions pertaining to food security, human and animal health, education, natural resource management and other vital activities. Local communities in developing countries in Africa are applying traditional medicine knowledge to respond to and manage the HIV and AIDS pandemic as well as in the treatment of other opportunistic infections and it forms an integral part of the culture and history of local communities and hence their common asset in their effort to gain control of their own lives.

Michika (Mwe-cika) town is the Head quarter of Michika local Government area of Adamawa state of Nigeria. It was located directly across the border from the famous tourist site of Rhumsiki in Cameroon. The principal ethnic group and language in Michika is the Kamue people and Kamue language. The local government was created in 1976. It is located in the northern axis of the state and is bordered on the east by the republic of Cameroon as shown in fig 1. On its north border is Madagali local government, while it shares border to the west by Askira Uba local government of Borno state, to the south by Mubi North and Hong local government. The word Michika is a corrupted word from Mwecika as reported by the oral history found around late $17^{\text {th }}$ century by Kwada Kwakaa a prince and a royal hunter. Mwe" in the Kamwe language means heaven, sky, hills, or even mountains used by relatives of a bereaved person on their waist and wrist to signify bereavement in connection of some medicinal plants for Protection against disease, ailment and spirit in the course of his duty [6].

Michika people are mainly farmers, hunters and women in fishing. As years pass they were good animals rearing, they rear domestic animals; pigs, cow, goats, and chickens. They are among the best educated community in Adamawa state due to early advents of Missionary Christian to the communities.

Kamwe belief in nature, spirits, ancestors and its connection with trees, stoned and mountains as part of power to heal and harm people and Animals. However, they are not worshipper before the coming of Christianity. There are some people who have the power to communicate with the spirits through media. They are notable and useful people among the communities. They are considered and act as seers and medicine men, claiming to use plants, stone and other means to solve the menace of life. The local government community recognized them as traditional healers because of their close association with trees which mostly grow throughout the years. They have learnt to utilize this resource for various ailment and diseases common among the communities. 


\section{Aim And ObJectives of The STUdy}

The study is aimed at surveying and documenting medicinal plants and their potentials in seven district; Bazza, Futu, Garta, Madzi, Michika, Nkafa, and Zah used in the treatment of disease and ailment in the local government.

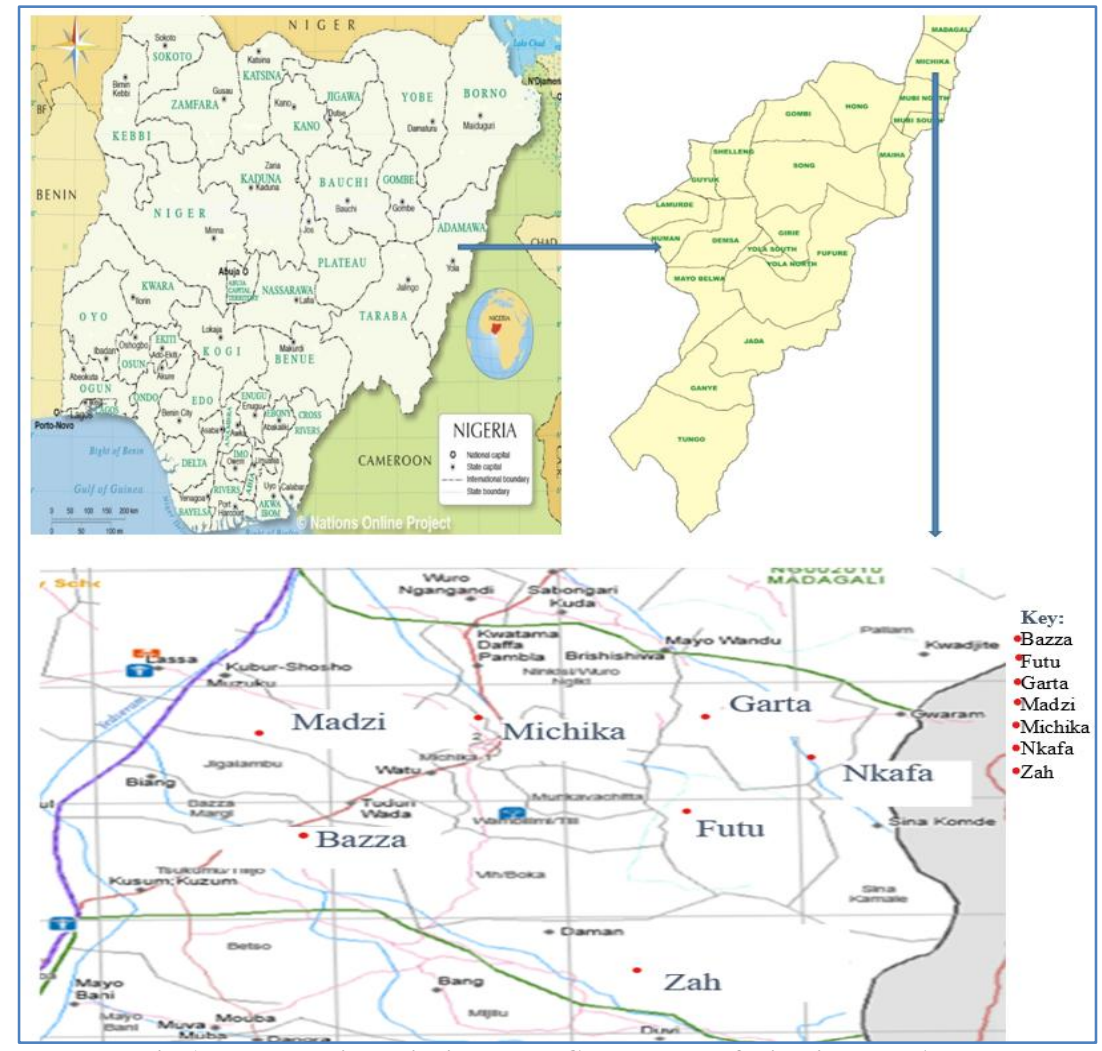

Fig-1: Map showing Michika Local Government of Nigeria study Area

\section{Methodology}

\section{Study Area}

The study was carried out in seven district of Michika Local Government Area of Adamawa State, Nigeria. The people have many medicinal tree plants surrounding them.

\section{Study Design}

Districts tours 48 to 50 days duration were planned to cover the seven districts to update the ethno medicinal plant species information. Two thousand one hundred informants were interviewed through questioners regarding the type of medicine plants used by them in their districts. Majority of the people that were interviewed include the full and part time herbalists, old ladies family heads and village heads in order to get a better understanding of the local customs, beliefs and habits.

\section{Medicinal tree Survey, Department of Biochemistry \\ University Wukari Nigeria \\ (One form should be completed for each tree) Age: Sex:}

1. Name:

2. Address

3. Occupation:

4. Local Nmae

\section{Stem-bark Characteristics}

7. Latex: Present Absent

8. Tree plants used in medicine

9. Roots: ___ Stem-bark

10. How a plant is used: Fresh: Twig: Dried:

Flowers: Fruits: seeds:

11. Other parts of tree and Ingredient added:

12. Method (s) of preparation for use: Powdered Water Extraction (cool): (Hot) Water extraction (boiled): Extraction with Argye (gin): Water extraction others: 


\section{Mode of administration:}

14. Dosage

15: Added Information

16. Community response on resource of Medicinal plant in the district:

Yes:

No:

Table-1: Demographic structure of respondents showing age range and sex

\begin{tabular}{|l|l|l|l|l|l|}
\hline Age range & $\begin{array}{l}\text { Number of } \\
\text { respondents }\end{array}$ & Male & $(\boldsymbol{\%})$ & Female & $(\boldsymbol{\%})$ \\
\hline $18-23$ & 180 & 120 & 66.7 & 60 & 33.33 \\
\hline $24-29$ & 300 & 180 & 60.0 & 120 & 40 \\
\hline $30-35$ & 350 & 250 & 71.43 & 100 & 28.57 \\
\hline $36-41$ & 350 & 230 & 65.71 & 120 & 34.29 \\
\hline $42-47$ & 410 & 300 & 73.17 & 110 & 26.83 \\
\hline $48-53$ & 300 & 150 & 50.0 & 150 & 50.0 \\
\hline $54-59$ & 310 & 210 & 67.74 & 100 & 47.62 \\
\hline $60-65$ & 300 & 100 & 33.33 & 200 & 66.66 \\
\hline
\end{tabular}

Table-2: Community response on resource and ranking of Medicinal plant in the seven districts

\begin{tabular}{|c|c|c|c|c|c|c|c|c|c|c|c|c|c|c|c|}
\hline \multirow{2}{*}{\multicolumn{2}{|c|}{ Medicinal plants }} & \multicolumn{14}{|c|}{ Districts of Michika Local Government } \\
\hline & & \multicolumn{2}{|c|}{ Bazza } & \multicolumn{2}{|c|}{ Futu } & \multicolumn{2}{|c|}{ Garta } & \multicolumn{2}{|c|}{ Madzi } & \multicolumn{2}{|c|}{ Michika } & \multicolumn{2}{|c|}{ Nkafa } & \multicolumn{2}{|c|}{ Zah } \\
\hline \multicolumn{2}{|l|}{ Ranking } & & 4 & & 3 & & 7 & & 5 & & 6 & & 1 & & 2 \\
\hline & & Yes & No & Yes & No & Yes & No & Yes & No & Yes & No & Yes & No & Yes & No \\
\hline 1. Acacia seyal & Skulye & 322 & 168 & 245 & 265 & 298 & 212 & 421 & 89 & 452 & 48 & 411 & 99 & 431 & 69 \\
\hline 2. Parka biglobosa & Lehune & 466 & 44 & 482 & 28 & 477 & 33 & 486 & 24 & 488 & 22 & 488 & 22 & 490 & 10 \\
\hline 3. Adonsonia digitata & Mithye & 499 & 1 & 498 & 2 & 499 & 1 & 467 & 33 & 484 & 16 & 491 & 9 & 496 & 4 \\
\hline 4. Khaya senegalensis & Chei & 388 & 112 & 464 & 56 & 488 & 22 & 456 & 44 & 388 & 112 & 490 & 10 & 497 & 3 \\
\hline 5. Ziziphus spina-christi & Kurnna & 364 & 136 & 477 & 23 & 480 & 20 & 432 & 68 & 333 & 167 & 476 & 24 & 485 & 15 \\
\hline 6. Azadirachta indica & Nemmei & 432 & 68 & 366 & 134 & 384 & 116 & 410 & 90 & 498 & 2 & 334 & 166 & 234 & 266 \\
\hline 7. Moringa oliefera & Tsaga (Galaganji) & 410 & 90 & 450 & 50 & 460 & 40 & 485 & 15 & 498 & 2 & 456 & 44 & 476 & 24 \\
\hline 8. Ficusgnaphalocarpa & Mukurahye & 372 & 128 & 410 & 90 & 400 & 100 & 442 & 58 & 438 & 62 & 411 & 89 & 388 & 12 \\
\hline 9. Mangifera indica & Magorea & 492 & 8 & 488 & 12 & 491 & 9 & 499 & 1 & 499 & 1 & 498 & 2 & 499 & 1 \\
\hline 10. Cassipourea congoensis & Twentwe & 123 & 377 & 344 & 156 & 112 & 388 & 67 & 453 & 12 & 488 & 338 & 62 & 403 & 97 \\
\hline 11. Carrissa macrocarpa & Ndre & 324 & 176 & 244 & 256 & 133 & 367 & 388 & 112 & 211 & 289 & 256 & 244 & 237 & 263 \\
\hline 12. Psidium guajava & Gwuavva & 477 & 23 & 488 & 12 & 489 & 11 & 476 & 24 & 489 & 11 & 492 & 8 & 494 & 6 \\
\hline 13. Vitex doniana & Shekai & 455 & 45 & 466 & 54 & 472 & 28 & 477 & 23 & 482 & 18 & 478 & 22 & 344 & 156 \\
\hline 14. Nuclea latifolia & Tanzhye & 23 & 477 & 345 & 155 & 34 & 466 & 12 & 488 & 10 & 490 & 435 & 65 & 344 & 156 \\
\hline 15. Haematostaphis barteri & Drunee & 123 & 377 & 312 & 188 & 144 & 356 & 12 & 488 & 11 & 482 & 342 & 158 & 398 & 102 \\
\hline 16. Mangifera indica & Mangoro & 456 & 44 & 455 & 45 & 488 & 12 & 486 & 14 & 490 & 10 & 496 & 4 & 466 & 34 \\
\hline 17. Anacardium occidental & Kasheuwe & 488 & 12 & 491 & 9 & 489 & 11 & 486 & 14 & 485 & 15 & 491 & 9 & 494 & 6 \\
\hline 18. Nuclea latifolia & Thyampye & 133 & 367 & 147 & 353 & 322 & 178 & 297 & 203 & 233 & 267 & 312 & 188 & 344 & 156 \\
\hline 19. Musa sapientum & Ayaba & 482 & 18 & 488 & 12 & 486 & 14 & 485 & 15 & 488 & 12 & 491 & 9 & 489 & 11 \\
\hline 20. Annona senegalensis & Dlye & 233 & 267 & 186 & 314 & 291 & 109 & 233 & 267 & 129 & 371 & 188 & 312 & 191 & 309 \\
\hline 21. Citrus aurrantium & Lamwue & 447 & 53 & 467 & 33 & 477 & 23 & 499 & 1 & 487 & 13 & 489 & 11 & 488 & 12 \\
\hline 22. Eucalyptus indica & Zaiti & 492 & 8 & 234 & 266 & 245 & 255 & 488 & 12 & 498 & 2 & 341 & 59 & 456 & 44 \\
\hline 23. Tamarindus indica & Mbulla & 499 & 1 & 498 & 2 & 498 & 2 & 499 & 1 & 499 & 1 & 500 & 0 & 500 & 0 \\
\hline 24. Vernonia amygdalina & Chuwaka & 344 & 156 & 133 & 367 & 112 & 388 & 244 & 256 & 456 & 44 & 84 & 418 & 12 & 488 \\
\hline 25. Cassia occidentalis & yelekachia & 441 & 59 & 344 & 156 & 234 & 266 & 299 & 211 & 137 & 363 & 122 & 378 & 156 & 344 \\
\hline 26. Phoenix dactylifera & Dibinowye & 12 & 488 & 11 & 499 & 8 & 492 & 3 & 497 & 3 & 497 & 12 & 377 & 12 & 488 \\
\hline 27. Citrus Paradise & Garepe & 344 & 146 & 388 & 112 & 456 & 44 & 387 & 113 & 466 & 34 & 399 & 101 & 411 & 89 \\
\hline 28. Jatropha curcas & Kwakwalajie & 237 & 263 & 311 & 189 & 222 & 278 & 231 & 269 & 322 & 178 & 244 & 256 & 239 & 261 \\
\hline 29.Momordica charantia & Muchika tyebere & 473 & 27 & 488 & 12 & 479 & 21 & 491 & 9 & 488 & 12 & 497 & 3 & 498 & 2 \\
\hline 30. Anogeissuss,leiocapus & Marke & 388 & 112 & 321 & 179 & 383 & 117 & 297 & 203 & 311 & 189 & 366 & 134 & 332 & 168 \\
\hline 31. Ficus sp & Baure & 459 & 41 & 466 & 34 & 474 & 26 & 477 & 23 & 486 & 14 & 483 & 17 & 458 & 42 \\
\hline 32. Ficus vollis & Ndree & 389 & 111 & 299 & 201 & 299 & 201 & 399 & 101 & 333 & 167 & 341 & 159 & 299 & 201 \\
\hline 33. Gemlin arborea & Melina & 334 & 168 & 329 & 171 & 364 & 138 & 322 & 178 & 299 & 201 & 277 & 223 & 312 & 188 \\
\hline 34. Borassus aethiopum & Dlye & 397 & 103 & 378 & 122 & 289 & 211 & 299 & 201 & 300 & 200 & 311 & 189 & 241 & 259 \\
\hline 35. Abrus precatorius & Ntse kwantikwu & 331 & 169 & 344 & 156 & 354 & 146 & 288 & 212 & 279 & 221 & 355 & 145 & 276 & 224 \\
\hline 36. Elaeophorbia drupifera & Hulie & 446 & 54 & 474 & 26 & 457 & 43 & 488 & 12 & 496 & 4 & 498 & 2 & 499 & 1 \\
\hline 37. Artemisia maciverae & Tazargade & 344 & 156 & 277 & 123 & 331 & 169 & 211 & 289 & 235 & 265 & 230 & 270 & 231 & 269 \\
\hline 38. Vitellaria paradoxa & Fuma & 488 & 12 & 498 & 2 & 497 & 3 & 489 & 11 & 499 & 1 & 500 & 0 & 500 & 0 \\
\hline 39. Zizipus Mauritania & Dyivi & 489 & 11 & 496 & 4 & 498 & 2 & 499 & 1 & 479 & 21 & 499 & 1 & 500 & 0 \\
\hline 40. Daniella oliverii & Lellekai & 455 & 45 & 460 & 40 & 489 & 11 & 476 & 24 & 493 & 7 & 492 & 8 & 499 & 1 \\
\hline 41. Anogeissus leiocarpa & Dyaka & 321 & 179 & 233 & 267 & 211 & 289 & 214 & 286 & 266 & 234 & 274 & 226 & 341 & 159 \\
\hline 42. Ficus sycomorus & Mabulme & 477 & 23 & 467 & 33 & 455 & 45 & 444 & 56 & 477 & 23 & 486 & 14 & 490 & 10 \\
\hline 43. Ficus asperifolia & Kwadachikwa & 357 & 143 & 331 & 169 & 349 & 151 & 299 & 201 & 331 & 169 & 367 & 123 & 388 & 112 \\
\hline 44. Sesamum Indicum & Malashirii & 456 & 44 & 467 & 33 & 457 & 43 & 488 & 12 & 487 & 13 & 490 & 10 & 496 & 4 \\
\hline 45. Leptadenia hastata & Yadiya & 477 & 23 & 487 & 13 & 488 & 12 & 488 & 12 & 499 & 1 & 498 & 2 & 498 & 2 \\
\hline
\end{tabular}




\begin{tabular}{|l|l|l|l|l|l|l|l|l|l|l|l|l|l|l|l|}
\hline 46. Conorandus panados & Mnizee & 233 & 267 & 244 & 256 & 256 & 244 & 277 & 223 & 234 & 276 & 320 & 280 & 379 & 121 \\
\hline 47. Deterium microcarpum & Tallow & 221 & 279 & 222 & 278 & 198 & 302 & 246 & 254 & 199 & 301 & 266 & 234 & 278 & 222 \\
\hline 48. Balanites aegytiaca & Betu (Jachamye) & 211 & 289 & 156 & 344 & 200 & 300 & 196 & 304 & 211 & 289 & 224 & 276 & 199 & 301 \\
\hline
\end{tabular}

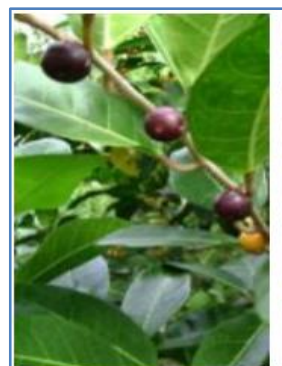

i. Ficus vollis (Ndree)

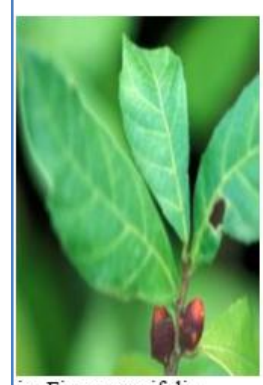

iv. Ficus asperifolia

Miquel, (Kwadachikwa)

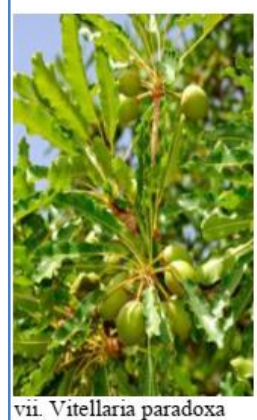

(Fuma)

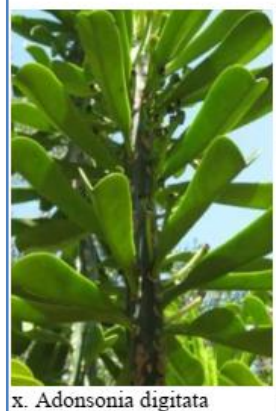

(Hulye)

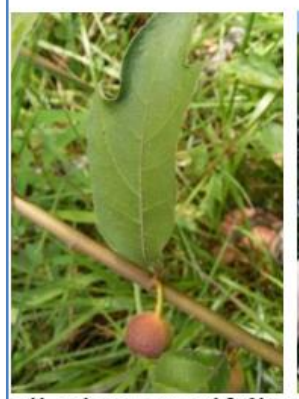

xii. Ficus asperifolia (Kwadachikwa)

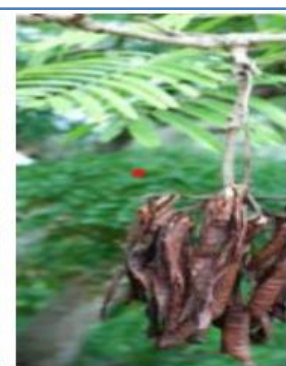

ii. Parkia biglobosa

(Lehune)

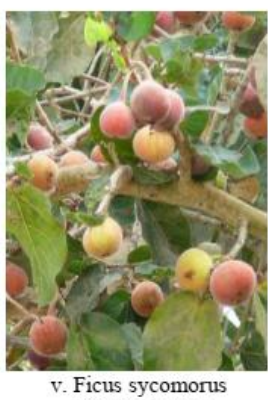

(Kwadachikwa)

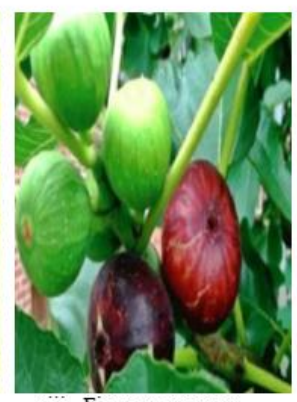

viii. Ficus sycomorus, (Mabulme)

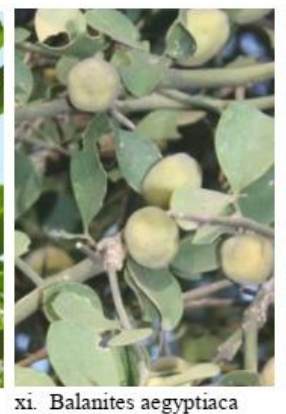

(Jachamye)

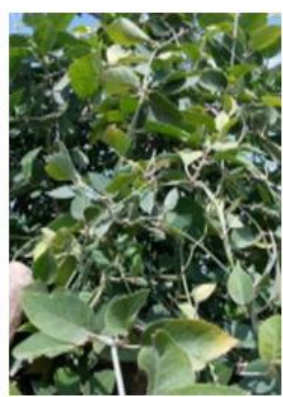

xiv. Leptadenia hastata (Yadiya)

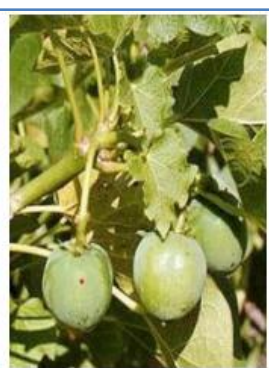

iii. Jatropha curcas (Kwakwalajie)

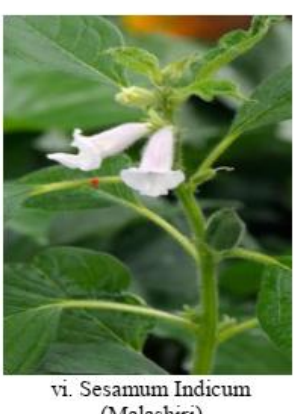
(Malashiri)

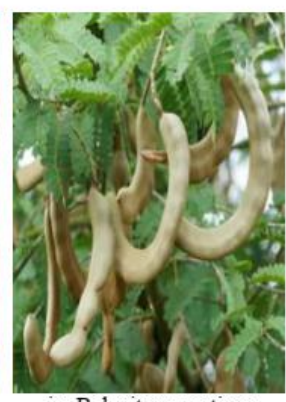

ix. Balanites aegytiaca (Mbulla)

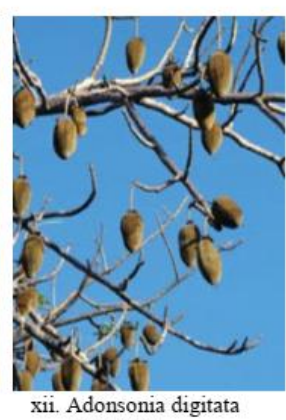

(Mythie)

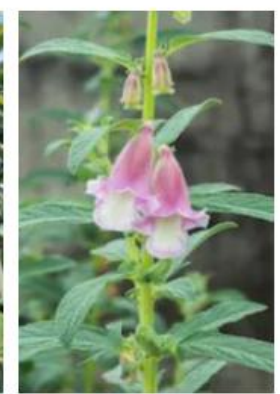

xv. Sesamum Indicum (Malashirii) 


\section{Fig-2: Some of the medicinal plant in Michika Local Government}

Table-3: Shows Medicinal plants, parts used and preparation

\begin{tabular}{|c|c|c|c|c|}
\hline Name of plant species & $\begin{array}{l}\text { Vernacular } \\
\text { Name }\end{array}$ & Parts used & Preparation & Usage \\
\hline 1. Acacia seyal & Skulye & Leaf, stem-bark, roots & Cool, boiling & Dysentery, malaria, ear drop \\
\hline 2. Parka biglobosa & Lehunie & Leaf, Stem-bark, roots & Boiling, decoctions & Malaria, worms, measles \\
\hline 3. Adonsonia digitata & Miethae & Leaf, stem-bark & Maceration & Placental retention \\
\hline 4. Khaya senegalensis & Chei & Seed, leaf, stem-bark, roots & Decoction & Stomach pain, with kunu \\
\hline 5. Ziziphus spina-christi & Kurnna & Leaf, stem-bark, roots & Maceration & Itching. Bacterial infection \\
\hline 6. Azadirachta indica & Nemmei & $\begin{array}{l}\text { Leafs, stem-bark, seeds, } \\
\text { and roots }\end{array}$ & Maceration, boiling & Malaria, fever, fungal, bacteria \\
\hline 7. Moringa oliefera & Galaganjie & $\begin{array}{l}\text { Leafs, roots, stem-bark, } \\
\text { seeds }\end{array}$ & Powder, maceration & Blood pressure, diabetic, dysentry \\
\hline 8. Ficusgnaphalocarpa & Mukurahye & Leaf, stem-bark & Boiling & After birth bath \\
\hline 9. Mangifera indica & Magorea & Leaf, stem-bark & Boiling, decoction & Stomach pain, fever, \\
\hline $\begin{array}{l}\text { 10. Cassipourea } \\
\text { congoensis }\end{array}$ & Twentwe & Leaf, stem-bark & Maceration & Inflammation \\
\hline 11. Carrissa macrocarpa & Ndre & Twits, leaf & Maceration, powder & Infectious disease, stomach pain \\
\hline 12. Psidium guajava & Gwuavva & Leaf & Decoction & Malaria, fever, inflammation \\
\hline 13. Vitex doniana & Shekai & Stem-bark, leaf & Decoction & Dysentery, worms, fever \\
\hline 14. Nuclea latifolia & Tanzhye & Stem-bark, leaf & Boiling, chewing & Tooth ache, stomach pain \\
\hline 15. Haematostaphis barteri & Drunee & Stem-bark, leafs, seeds & Boiling, powder & Stomach pain, inflammation, \\
\hline 16. Mangifera indica & Mangoro & Stem-bark, leaf & Maceration & Wounding healing, inflammation \\
\hline 17. Anacardium occidental & Kasheuwe & Leaf, stem-bark & Decoction & Malaria, stomach pain \\
\hline 18. Nuclea latifolia & Thyampye & Leaf, stem-bark, roots & Boiling & Inflammation, hypertension, fever \\
\hline 19. Musa sapientum & Ayaba & Leaf & Maceration & Stomach pain \\
\hline 20. Annona senegalensis & Dthye & Leaf & Decoction & Inflammation, dysentery \\
\hline 21. Citrus aurrantium & Lamwue & Leaf, stem-bark & Decoction & Malaria, fever, inflammation \\
\hline 22. Eucalyptus indica & Zaiti & Leaf, stem-bark & Maceration & Catarrh, Inflammation \\
\hline 23. Tamarindus indica & Mbwula & Leaf, stem-bark & Boiling, Maceration & $\begin{array}{l}\text { Blood pressure, Join pain, } \\
\text { Diabetes }\end{array}$ \\
\hline 24. Vernonia amygdalina & Chuwaka & Leaf & Maceration & Diabetes, stomach pain, fever \\
\hline 25. Cassia occidentalis & Yelekachia & Leaf, stem-bark & Boiling & Yellow fever, malaria \\
\hline 26. Phoenix dactylifera & Dibinowye & Fruits & Chewing & Stomach pain, dysentery \\
\hline 27. Citrus Paradise & Garepe & Leaf & Boiling & Malaria, headache \\
\hline 28. Jatropha curcas & Kwakwalajie & Leaf, sap, stem-bark & $\begin{array}{l}\text { Maceration, } \\
\text { secretion }\end{array}$ & $\begin{array}{l}\text { Wound healing, diabetic and } \\
\text { ulcer }\end{array}$ \\
\hline 29.Momordica charantia & Muchika tyebere & Leaf, stem-bark & Boiling, maceration & Stomach pain, fever, food \\
\hline 30. Anogeissuss, leiocapus & Marke & Stem-bark & Powder & skin infections \\
\hline 31. Ficus sp & Baure & Leaf, stem-bark & Leaf, stem-bark & Stomach pain, Headache \\
\hline 32. Ficus vollis & Ndree & Leaf, stem-bark & Leaf & Running nose \\
\hline 33. Gemlin arborea & Melina & Leaf, stem-bark roots & Maceration & Infection diseases, skin, throat, \\
\hline 34. Borassus aethiopum & Dlye & Leaf stem-bark, roots & Boiling, powder & $\begin{array}{l}\text { Stomach pain, fever, } \\
\text { inflammation }\end{array}$ \\
\hline 35. Abrus precatorius & Ntse kwantikwu & Leaf stem-bark, roots & Boiling & Bacterial, fungi, diabetes \\
\hline 36. Elaeophorbia drupifera & Hulie & Leaf, sap & Maceration & Infection diseases skin \\
\hline 37. Artemisia maciverae & Tazargade & Leaf, stem-bark, roots & $\begin{array}{l}\text { Decoction, } \\
\text { Maceration }\end{array}$ & Antiparasites, stomach pain \\
\hline 38. Vitellaria paradoxa & Fuma & Leaf, stem-bark, roots & Maceration & stomach ache, headaches, fever \\
\hline 39. Zizipus Mauritania & Dyivi & Leaf, stem-bark & Boiling & $\begin{array}{l}\text { Blood pressure, cholesterol, } \\
\text { anemia. }\end{array}$ \\
\hline 40. Daniella oliverii & Lellekai & Twist, leaf & Decoction & Bacterial, fungi, diabetes, fatigue \\
\hline 41. Anogeissus leiocarpa & Dyaka & Leaf, stem-bark, roots & Maceration, boiling & $\begin{array}{l}\text { Antibacterial, antifungal, stomach } \\
\text { pain }\end{array}$ \\
\hline 42. Ficus sycomorus & Kwadachikwa & Leaf, stem-bark & Maceration, powder & $\begin{array}{l}\text { Cardiovascular, potency, stomach } \\
\text { pain }\end{array}$ \\
\hline 43. Ficus asperifolia & Mabulme & Leaf, stem-bark, roots & Boiling, powder & Wound healing, diabetes. \\
\hline 44. Sesamum Indicum & Malashirii & Seed, leafs and roots & Maceration & Infection, Constipation \\
\hline 45. Leptadenia hastata & Yadiya & Stem-bark, leaf & Boiling, Maceration & $\begin{array}{l}\text { Inflammation, ulcer, Blood } \\
\text { pressure }\end{array}$ \\
\hline 46. Conorandus panados & Mnizee & Leaf, stem-bark & Decoction & Inflammation, headache \\
\hline $\begin{array}{l}\text { 47. Deterium } \\
\text { microcarpum }\end{array}$ & Tallow & Stem-bark & Decoction & Stomach ache, inflammation \\
\hline 48.Balanites aegytiaca & Betu & Stem-bark, leaf & Boiling, Maceration & Inflammation, wound healing, \\
\hline
\end{tabular}




\begin{tabular}{|l|l|l|l|l|}
\hline & & & ulcer \\
\hline 49. Carica papaya & Mbuposari & Seed, leaf, stem-bark, root & Boiling, Maceration & $\begin{array}{l}\text { Infection, diabetes, blood } \\
\text { pressure }\end{array}$ \\
\hline
\end{tabular}

\section{RESUlT AND DisCUSSION}

The survey of the ethno medicinal account of 49 plants species as shown in Table 2 and Table 3 with their local names, followed by plants part mode of administration and usage were enumerated. Demographic structure of respondents showing age range and sex were also taken into consideration, in the survey, the herbal practitioner, full and part time herbalists, old ladies, family heads and village heads participated in the survey in order to get a better understanding of the local customs, beliefs, usage and habitat of the medicinal plants. The plants mentioned indicated how rich Michika is in plant diversity, which are used among the district communities of the local government. Kamwue for their daily treatment of diseases, ailment and economical values. Kamwue people enjoy a good reputation in the traditional medicine, despite the efforts of the researcher to intensify modern health practice and the use of synthesized drugs, traditional healers are still playing their parts as the only medication that is potent, cheap and affordable by the less privileged people among the communities living in the remote part of the local government area. These plants are mostly used as medicine and as vegetables.

Table 2 shows the report of respondent of their knowledge of the medicinal plants by indicating yes if you know and have the knowledge of the medicinal plant and No if there is no knowledge at all. With results obtained 85-90\% showed a good knowledge of the medicinal plant which resulted to the significant of this research work.

Table 3. Shows the medicinal value as potential agents for treatment of many diseases such as malaria, fever, headache, dysentery, hypertension, diabetes etc. And because some of this plants are seasonal, the resources can be harnessed for the pharmacological investigation by the researcher to be prepared in the form of agents to impade the menace of resistance pathogen and terminal diseases such as cancer, ulcer, diabetic, hypertension and the present pandemic Corona virus (COVID-19).

Figure 2 shows some of the medicinal plant obtained in the seven district of Michika local Government of Nigeria, which are found to halt the diseases and ailment that has been troubling the communities' right from ages. These plants have been used and pass to from generation to regenerations till this day.

\section{CONCLUSION}

The survey has listed some of the medicinal plants obtained among the community of Michika local government. The plants listed was reported by the respondents to be safe from ages as per the recommendation of the traditional healer, and part time herbalists, old ladies, family heads and village heads who participated in the survey. The survey also suggested that some of this plants which are used as ethno-medically throughout the local government to cure various diseases and ailment could be exploited for new agents for many diseases which has become resistance and a menace to the host. From the survey and the respondent shows high evidence of efficacy of the medicinal plants

\section{RECOMMENDATION}

The major challenges to the use of medicinal plants to treat diseases and ailment is the lack of evidence of the standard required dosage of the remedies, established evidence of its potency, safety and significant certainty of the mechanism. Therefore, guidance should be created to give a better understanding of evaluating the dosage, safety, and significance of this medicinal plants, to enable fight the resistance diseases as result of synthesized drugs. we also recommend that a detailed ecological studies should be carried out within the district and other reserved areas in the local government to know their biodiversity status which will later serve as a guide to formulate a better policy and law to protect the extinct of medicinal plants.

\section{ACKNOWLEDGEMENT}

We are greatly indebted to Federal University Wukari, District heads, traditional herbalist, part time healers and the community at large for the full cooperation given to our teams.

\section{Declaration of conflicting interests}

The authors declared no potential conflicts of interest with respect to the research, authorship as well as the publications of this research work.

\section{Funding}

The authors received no financial support for the research, authorship and publication of this research work 


\section{REFERENCE}

1. World Health Organization. (2001). Legal status of traditional medicine and complementary (No. WHO/EDM/TRM/2001.2). Geneva: World Health Organization.

2. Sithole, J. (2007). The challenges faced by African libraries and information centres in documenting and preserving indigenous knowledge. IFLA journal, 33(2), 117-123.

3. Idu, M. (2009). The plant called medicine. An inaugural lecture of the University of Benin delivered by Professor MacDonald Idu on February 5, 2009). Guardian March 16, 2009.

4. Okoli, R. I., Aigbe, O., Ohaju-Obodo, J. O., \& Mensah, J. K. (2007). Medicinal herbs used for managing some common ailments among Esan people of Edo State, Nigeria. Pakistan Journal of Nutrition, 6(5), 490-496.

5. Nijar, G. S. (1996). TRIPS and Biodiversity: The Threat and Responses: A Third World View (Vol. 2). Third World Network.

6. Kwache, Iliya, Y. (2016). Kamwe People of Northern Nigeria: Origin, History and Culture (1st Ed.). Kaduna, Nigeria: Prudent Publishers. 